\title{
Treating Oral Leukoplakia with a 532-nm Pulsed Diode Laser
}

Nu-Ri Im ${ }^{1}$
Byoungjae Kim,2
Jian Kim¹
Seung-Kuk Baek ${ }^{1}$

${ }^{1}$ Department of Otorhinolaryngology-Head and Neck Surgery, Korea University College of Medicine, Seoul, Korea

${ }^{2}$ Neuroscience research institute, Korea University College of Medicine, Seoul, Korea
Received June 26, 2019

Accepted June 27, 2019

\section{Correspondence}

Seung-Kuk Baek

Department of Otorhinolaryngology-Head and Neck Surgery, Korea University Anam Hospital, 73 Inchon-ro, Seongbuk-gu, Seoul 02841, Korea Tel.: +82-2-920-6618

Fax: $+82-2-920-5233$

E-mail: mdskbaek@gmail.com

(C) Korean Society for Laser Medicine and Surgery

(c) This is an open access article distributed under the terms of the Creative Commons Attribution NonCommercial License (http://creativecommons.org/ licenses/by-nc/4.0) which permits unrestricted noncommercial use, distribution, and reproduction in any medium, provided the original work is properly cited.
A 50-year-old Korean female with oral erythro-leukoplakia was treated using a 532-nm Diode laser at 6 Watts with a pulsed width of 25 milliseconds. After two months following the laser treatment, the resected region was well-healed without any significant scar contracture. We suggest that the use of the 532-nm Diode laser can be a safe and effective treatment modality for patients suffering from oral leukoplakia.

\section{Key words}

Laser; Erythroplakia; Leukoplakia 


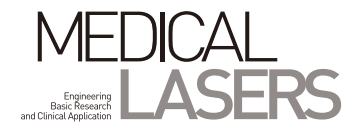

\section{INTRODUCTION}

Oral leukoplakia that is defined as a white patch or plaque of the oral mucosa that cannot be characterized clinically or pathologically as any other disease.' Clinically, these lesions are associated with malignant transformation. Almost $50 \%$ of all oral squamous cell carcinomas are preceded by oral leukoplakia as premalignant lesions. ${ }^{2}$ The previous studies have reported the percentage of malignant transformation of leukoplakia lesions as $7.6 \%$ to $17.5 \%{ }^{3,4}$ Various nonsurgical treatment modalities for oral leukoplakia have been reported, but they have shown a disadvantage of high relapse after treatmentdiscontinuing. 5.6 Surgical treatment for oral leukoplakia can be performed with various tools including cold knife, cryosurgery, electrocoagulation, and laser. Since the first description on $\mathrm{CO}_{2}$ laser surgery for tongue lesions, Lasers using many different wavelengths have been used in the treatment of oral leukoplakia. ${ }^{7}$ As the advantages of laser surgery for oral lesions, Surgeons have excellent visibility during the operation by controlling bleeding and processes of wound healing are excellent, because of the limited scarring and contraction. ${ }^{7}$

Recently, clinical applications of 585-nm Pulse-Dye Laser (PDL) and 532-nm Potassium-Titanyl-Phosphate (KTP) lasers for laryngeal Reinke's edema have reported. ${ }^{8,9}$ These two lasers with the similar wavelength are angiolytic laser that was developed based on the concept of selective photothermolysis, which allows for photocoagulation of microvascular lesions and minimal injury of the surrounding tissues.

Therefore, we report a case of oral leukoplakia that was successfully treated by the 532-nm Diode laser.

\section{CASE REPORT}

A 50-year-old female presented with a 1-year history of erythro-leukoplakia on right buccal region. The chief complaint was a painful buccal lesion with shallow ulceration (Fig. 1A). The patient had no prior history of systemic disease such as diabetes mellitus and hypertension and also trauma or irritation on buccal region that may be elicited by dentures. She was a social drinker and a smoker who smoked a half pack of cigarettes a day for 20 years. There was no possible cause in hematologic examinations and in bacterial/fungal culture tests of oral mucosa. The pathologic result of punch biopsy for the lesion showed an acute and chronic inflammation. Thus, she underwent various treatments including oral steroid medications or intralesional steroid injections, but the lesion was not alleviated.

We decided to treat the erythro-leukoplakia with the 532-nm Diode laser (QUANTA D-8 ${ }^{\circledR}$, Quanta System S.p.A. Solbiate Olona, Italy). After general anesthesia, the buccal lesion was exposed using a mouth gag and was stained with Lugol's iodine solution to well-identify the margins of the buccal erythro-leukoplakia (Fig. 1B). The unstained and whitish lesion was irradiated with the 532-nm laser set at 6 Watts with a pulsed width of 25 milliseconds. The treatment end point was visualized blanching of the buccal mucosa at the time of laser treatment, which is consistent with ischemia secondary to photoablation of vessels of the irradiated lesion (Fig. 1C). Then, the irradiated lesion was separated with a cotton ball along the cleavage plane that was created following treatment with the 532$\mathrm{nm}$ laser (Fig. 1D).

The H\&E staining of the specimen showed a clear sep-

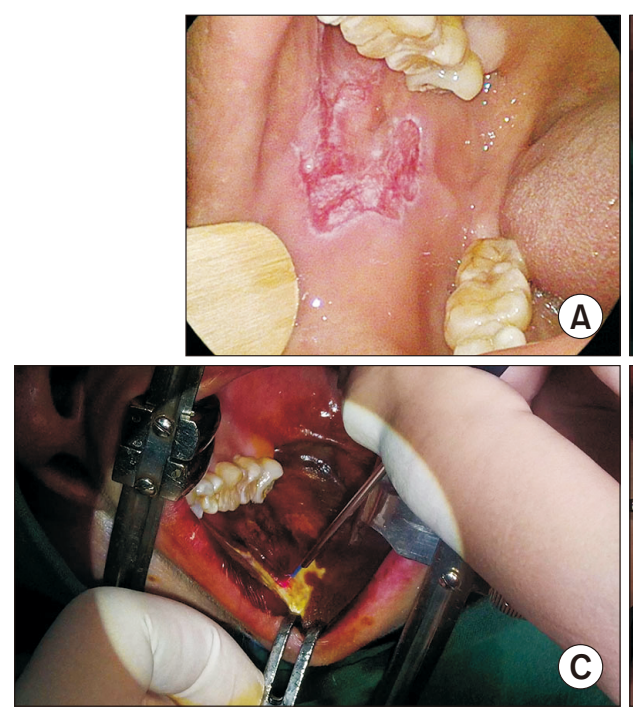

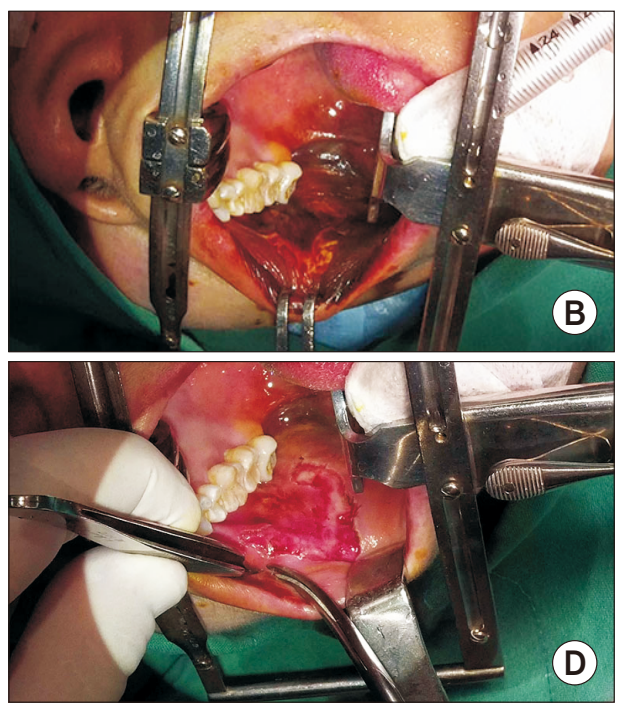

(D)
Fig. 1. The preoperative and intraoperative findings of oral erythroleukoplakia. (A) Erythro-leukoplakia lesion of right buccal mucosa. (B) After Lugol's iodine solution, the pathologic lesion is not stained and is well-identified. (C) The unstained and whitish lesion is irradiated with the 532-nm Diode laser and then the irradiated buccal mucosa blanches. (D) After removal of the lesion. 

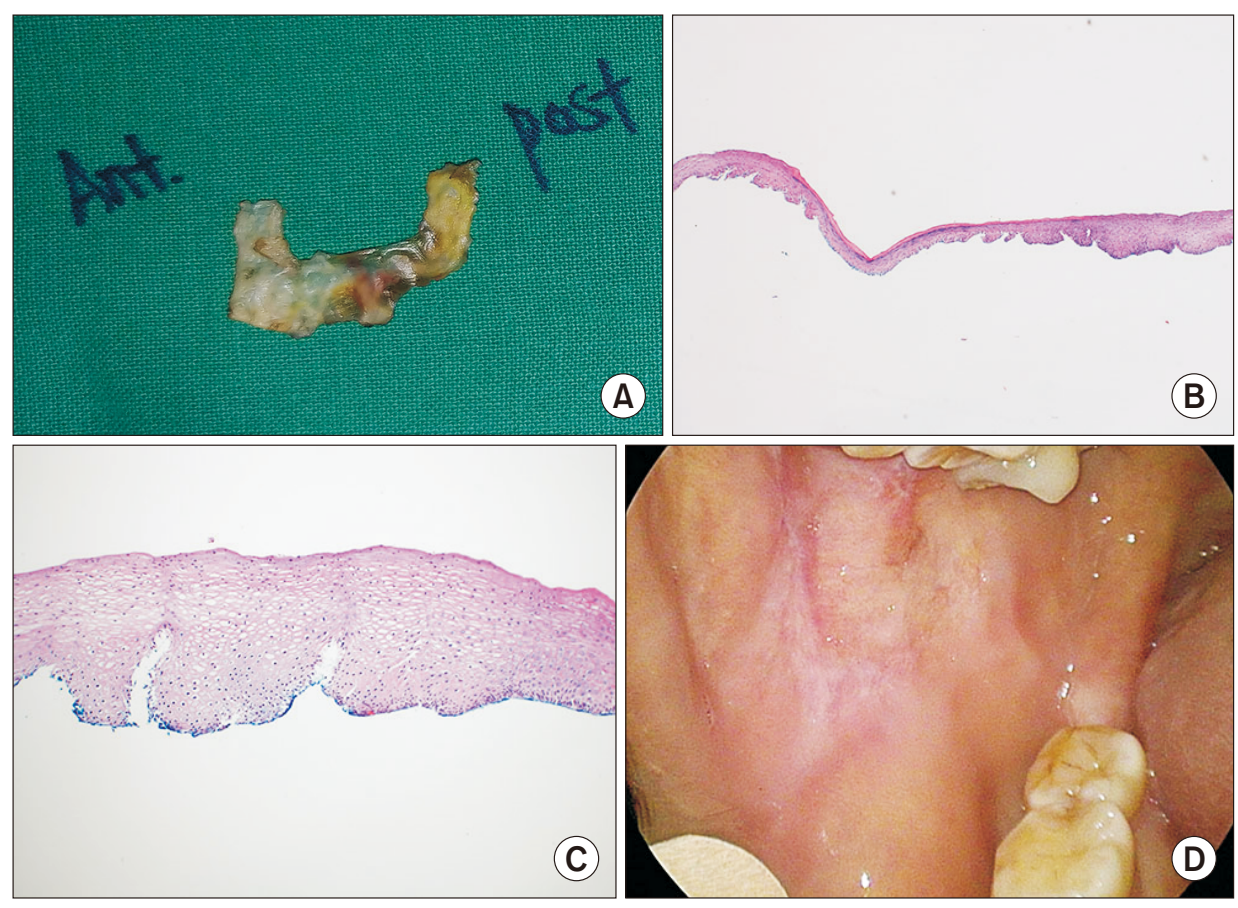

Fig. 2. The specimen and the final postoperative finding. (A) The resected specimen. $(\mathrm{B}, \mathrm{C})$ The $\mathrm{H} \& \mathrm{E}$ staining of the specimen shows a clear separation around the basement membrane between mucosal and submucosal layers. (D) The buccal region in postoperative 2 months following the operation. aration around the basement membrane between mucosal and submucosal layers (Fig. A-C). The final pathologic result was reported as chronic submucosal inflammation. Two months following the operation, the patient's buccal lesion demonstrated well-healed and normal mucosa without erythro-leukoplakia (Fig. 2D).

\section{DISCUSSION}

Early diagnosis of oral cavity cancer is one of critical issues to get good treatment outcome. Therefore, the management of oral leukoplakia that is considered as a premalignant lesion of oral mucosa is clinically very important. In addition, since there may be recurrence and/ or malignant transformation irrespective of the treatment procedure, it is very important to control oral leukoplakia in the management of oral cancer. The advantages of the laser such as hemostatic effect, precise resection, and minimal collateral damage have rendered the laser to be applied in the surgical resection of oral leukoplakia. ${ }^{10-12}$

Although $\mathrm{CO}_{2}$ laser excision have traditionally been applied to the treatment of oral leukoplakia, it may be difficult to minimize injury to the submucosal region during the procedure despite careful operation. Therefore, the application of 585-nm PDL to benign vocal cord disease have been reported and suggested that PDL is superior to CO2 laser. 8,13 The mechanism to remove a tissue with the PDL is photocoagulation of submucosal microcircula- tion. In particular, PDL denatures basement membrane anchoring fibers located in the lamina lucida of the basement membrane through a photoacoustic or photothermal effect and creates a cleavage plane between the epithelium and superficial lamina propria. ${ }^{14}$

The 532-nm KTP laser has the similar wavelength to PDL and is angiolytic laser that was developed photocoagulation of microvasculatures within submucosal area. The previous study has reported that the KTP laser may be more effective than the $\mathrm{CO}_{2}$ laser in preventing recurrences of oral leukoplakia because of its greater tissue penetrance and larger thermal scatter. ${ }^{10}$ However, since the KTP lasers were used to excise, and not ablate, lesions, the photocoagulation effect of the KTP laser have not been used in the past studies.

In the present case, after irradiation of the 532-nm Diode laser on oral leukoplakia, the cleavage between mucosal layer and submucosal layer was easily elicited. The cleavage plane allows the surgeon to perform the en-bloc excision by using cold instruments precisely and easily. After operation, the mucosa was healed without severe pain and scar contracture.

\section{CONCLUSIONS}

The 532-nm Diode laser excision may be a useful technique to remove oral leukoplakia with minimal collateral tissue damage through the photocoagulation of micro- 
vascular structure around basement membrane.

\section{CONFLICTS OF INTEREST}

The authors declare no conflicts of interest.

\section{FINANCIAL DISCLOSURE}

This research was supported by the Basic Science Research Program through the National Research Foundation of Korea funded by the Ministry of Education (NRF2016R1D1A1A02937362, NRF-2018R1D1A1A09083263).

\section{REFERENCES}

1. Kramer IR, Lucas RB, Pindborg JJ, Sobin LH. Definition of leukoplakia and related lesions: an aid to studies on oral precancer. Oral Surg Oral Med Oral Pathol 1978;46:518-39.

2. Schepman KP, van der Meij EH, Smeele LE, van der Waal I. Malignant transformation of oral leukoplakia: a follow-up study of a hospital-based population of 166 patients with oral leukoplakia from The Netherlands. Oral Oncol 1998;34:270-5.

3. Waldron CA, Shafer WG. Leukoplakia revisited. A clinicopathologic study 3256 oral leukoplakias. Cancer 1975;36:1386-92.

4. Silverman S Jr, Gorsky M, Lozada F. Oral leukoplakia and malignant transformation. A follow-up study of 257 patients. Cancer 1984;53:563-8.

5. Epstein JB, Wong FL, Millner A, Le ND. Topical bleomycin treatment of oral leukoplakia: a randomized double-blind clinical trial. Head Neck 1994;16:539-44.

6. Sankaranarayanan R, Mathew B, Varghese C, Sudhakaran PR, Menon V, Jayadeep A, et al. Chemoprevention of oral leukoplakia with vitamin A and beta carotene: an assessment. Oral Oncol 1997;33:231-6.

7. Ishii J, Fujita K, Komori T. Laser surgery as a treatment for oral leukoplakia. Oral Oncol 2003;39:759-69.

8. Park YM, Jo KH, Hong HJ, Choi HS. Phonatory outcome of 585 $\mathrm{nm} /$ pulsed-dye laser in the management of glottic leukoplakia. Auris Nasus Larynx 2014;41:459-63.

9. Pitman MJ, Lebowitz-Cooper A, lacob C, Tan M. Effect of the $532 \mathrm{~nm}$ pulsed KTP laser in the treatment of Reinke's edema. Laryngoscope 2012;122:2786-92.

10. Lim B, Smith A, Chandu A. Treatment of oral leukoplakia with carbon dioxide and potassium-titanyl-phosphate lasers: a comparison. J Oral Maxillofac Surg 2010;68:597-601.

11. Gáspár $L$. The use of high-power lasers in oral surgery. J Clin Laser Med Surg 1994;12:281-5.

12. Gendelman H, Actis AB, Ouri HO. Neodymium-YAG and CO2 lasers in treatment of pre-cancerous lesions of the oral cavity. Acta Stomatol Belg 1993;90:95-101.

13. Hwang CS, Lee HJ, Ha JG, Cho Cl, Kim NH, Hong HJ, et al. Use of pulsed dye laser in the treatment of sulcus vocalis. Otolaryngol Head Neck Surg 2013;148:804-9.

14. Franco RA Jr, Zeitels SM, Farinelli WA, Anderson RR. 585-nm pulsed dye laser treatment of glottal papillomatosis. Ann Otol Rhinol Laryngol 2002;111:486-92. 